\title{
Effect of didactic educational intervention on improving knowledge of antibiotics use and resistance in Yogyakarta Community
}

\author{
Susi Ari Kristina ${ }^{*}$, \\ Nada Nisrina Salsabila², \\ Yulianto Yulianto ${ }^{3}$, \\ Gerhard Fortwengel ${ }^{4}$ \\ ${ }_{1}$ Department of Pharmaceutics, Faculty \\ of Pharmacy, Universitas Gadjah Mada, \\ Yogyakarta, Indonesia \\ 2 Research Assistant, Faculty of Pharmacy, \\ Universitas Gadjah Mada, Yogyakarta, \\ Indonesia \\ 3 Indonesian Pharmacists Association, \\ Yogyakarta Province, Indonesia \\ ${ }^{4}$ Department of Media, Information and \\ Design, University of Applied Sciences and \\ Arts, Hannover, Germany
}

\section{*Corresponding author:}

Susi Ari Kristina

susiari_k@ugm.ac.id

\section{Keywords:}

Educational intervention,

Pharmacists, Knowledge, Antibiotic resistance

\begin{abstract}
Lack of knowledge regarding antibiotics use has been widely identified as a main reason for inappropriate antibiotics use which leads to antibiotic resistance phenomenon. This study aimed to evaluate the effects of pharmacist-initiated educational intervention on promoting appropriate use of antibiotics and reducing self-medication with antibiotics. A pre and post intervention study using two validated self-administered questionnaires was performed in Yogyakarta province. A-two hour session of course and case discussion was delivered as method of intervention. Pharmacy customers attended Gema Cermat program were invited conveniently to complete both of pre- and posteducational questionnaires. Descriptive presentation was conducted to show scores on questions. Knowledge scores were categorized as poor, adequate and high. Of 268 respondents, $34.22 \%$ respondents had poor level of knowledge before receiving educational intervention, but this number decreased into $12.21 \%$ after post-interventional phase. Another $28.23 \%$ respondents had adequate level of knowledge before and then elevated into $38.28 \%$ after receiving education about appropriate use of antibiotics. Pre-education, $37.43 \%$ participants had a high level of knowledge about antibiotics use and resistance, whereas after education the number became slightly higher (49.25\%). A vast majority of respondents (75.24\%) became more aware about appropriate antibiotics practice after receiving educational intervention. Overall, didactic educational intervention imposed higher knowledge and better practice regarding antibiotics use $(p<0.05)$. This study showed that using didactical education intervention towards antibiotics use and resistance can be an initial strategy that led to substantial improvement of appropriate antibiotics use. Further systemic interventions to educate people should be performed and evaluated in order to promote the appropriate use of antibiotics.
\end{abstract}

\section{INTRODUCTION}

A worldwide threat called antibiotic resistance is now alarming to take us back to a pre-antibiotic era as a big consequence of inappropriate use of antibiotics ${ }^{1}$. Antibiotic resistance is estimated to be a primary cause of 10 million deaths in 2050 according to the World Health Organization data ${ }^{2}$. Misuse and overuse of antibiotics had been acknowledged as a factor that accelerates the acquisition of resistance despite the fact that antibiotic resistance is actually a natural phenomenon ${ }^{3,4}$. Many factors including lack of knowledge
Pharmaceutical Sciences Asia (C) 2021 by Faculty of Pharmacy, Mahidol University, Thailand is licensed under CCBY-NC-ND 4.0. To view a copy of this license, visit https:// www.creativecommons.org/ licenses/by-nc-nd/4.0 
and concern, underestimation of antibiotic resistance, patients' expectations for antibiotics followed by the pressure on physicians about these expectations, had been identified as complex factors which highly influence antibiotic resistance phenomenon ${ }^{5-7}$.

In order to minimize antibiotic resistance phenomenon, the first thing to do is aimed to minimize inappropriate use of antibiotics. Evidence suggests that antibiotic stewardship programs (ASP) can decrease overuse of antibiotics, curtail antibiotic-associated events, reduce antibiotic resistance, and improve patient outcomes ${ }^{8,9}$. One of ASP came in form of antibiotics awareness campaign to promote the optimal use of antibiotics. Several studies suggest that public campaign through written material and mass media strengthen by healthcare professionals' role in educating patient could be more effective in promoting appropriate use of antibiotics ${ }^{10,11}$.

Antibiotic resistance in Indonesia is estimated to be high despite the limited data on antibiotic-related study ${ }^{12}$. This phenomenon was supported by publics' behavior in self-medication with antibiotics. In eastern of Indonesia, probability of self-medication with antibiotics tend to be done by people with lower knowledge on antibiotics $^{13}$. In Yogyakarta city, inappropriate use of antibiotics was also found as a result of publics' limited knowledge and attention in antibiotics indication which actually cannot be used to treat viral infections and prevent other diseases ${ }^{14}$. Our latest work reported that over $50 \%$ of the study population had a low level of awareness towards antibiotics use and resistance, and do not really consider antibiotic resistance as one of biggest problems the world faces ${ }^{15}$. These portraits show that interventions such as public campaign or any other form of education are needed to change publics' incorrect behavior related to antibiotics use.

Only few research data about the impact of educational intervention on increasing publics' knowledge and practice regarding antibiotics in Indonesia. Therefore, it is very important to evaluate whether educational intervention can be an effecttive strategy implemented in this country to promote appropriate antibiotics use. In our first study, the role of pharmacists as the source of information about antibiotics use and resistance was mentioned by $58 \%$ of the respondents and that result demonstrated the critical role of pharmacists in raising public awareness through several types of intervention ${ }^{16}$. Hence, the present study aims to assess the effects of pharmacist-initiated educational intervention on promoting appropriate antibiotics use and minimize self-medication with antibiotics.

\section{MATERIALS AND METHODS}

A quasi-experimental study design was used. A structured pre- and post-educational intervention delivered by pharmacists was conducted in May 2019. Adults residents living in Yogyakarta were invited to join the course held by Gema Cermat Pharmacists team. Sample respondent were conveniently selected based on willingness to participate in this study. Courses were consisted of two-hour didactic method and case presentation. A validated questionnaires was used to assess publics' knowledge and practice towards antibiotics use. The self-administered questionnaires were developed by reviewing several previous relevant published studies. Methods and content of education were face validated by practicing pharmacists considered as experts in social pharmacy. Content validity index was used to assess relevancy of items with construct of module. A pilot study on was carried out on 10 non-sample respondents live in Yogyakarta province.

The pre- and post- educational questionnaire consisted of 2 parts assessed participants' knowledge on antibiotics use and resistance and also practice of antibiotic usage. The first set of questionnaires concerned respondents' knowledge of antibiotics on the basis of indication, access, administration, and resistance to antibiotics. Participants were asked if each of eleven statements about antibiotics were 'true' or 'false'. The statements were as follows: (1) Antibiotics work on most cold and cough; (2) Antibiotics can treat a sore throat; (3) Antibiotics can kill bacteria; (4) Antibiotics can be used to treat viral infections; (5) Antibiotics can be purchased without prescription; (6) Leftover antibiotics are good to keep at home in case of future need; (7) If you feel better you can stop taking antibiotics; (8) Taking less antibiotic than prescribed is healthier than taking the full course; (9) Overuse of antibiotics can cause antibiotic resistance; (10) Antibiotic resistance occurs when the body becomes resistant to antibiotics; (11) Antibiotic resistance is only a problem for people who take antibiotics regularly. The second set of questions asked the respondents about their practice of antibiotics usage to determine their appropriate practice in antibiotics use. Respondents were asked whether they agree or disagree about four statements regarding their practice towards antibiotics as 
follows: (1) I am taking antibiotics only for infection; (2) I got antibiotics from prescription; (3) I am taking full dose of antibiotics prescribed; (4) I have become more cautious about antibiotics use. Pretest was conducted an hour before intervention, while posttest was assigned to the same participants at two weeks after intervention. These timeframe was chosen to avoid recall bias of respondent. Two weeks is considered ideal time gap for intervention study to assess the effectiveness. This study protocol was approved by Medical and Health Research Ethics Committee, Universitas Gadjah Mada, Yogyakarta.

Descriptive analysis was performed to describe the data in the form of frequency table. Correct responses in each statement were used to calculate respondents' knowledge and practice. The results of this study were presented in a form of charts and table to describe the effect of intervention on participants' knowledge and practice improvement. The difference between pre-post intervention was assessed by Wilcoxon rank test.

\section{RESULTS}

A total of 268 participants completed the pre- and post-intervention questionnaires.
The socio-demographic characteristics of respondents are indicated a high percentage of female participants (65\%), aged $25-45$ years $(72 \%)$, live in urban areas (67.2\%), passed a bachelor degree $(64 \%)$, and monthly income not more than 2 million IDR (51\%). Majority of respondents admitted that their source of antibiotics information was derived from pharmacists $(68 \%)$. More than half $(56 \%)$ of the participants had taken antibiotics in previous three months.

Knowledge scores was examined through the correct responses in the first area of survey. The correct responses by participants for each statement in the pre- and post-intervention questionnaires are presented in Table 1.

The level of knowledge on antibiotics use and resistance before and after education was assessed in each domain of questions including indication of antibiotics, access of antibiotics administration of antibiotics and also antibiotic resistance. Pre-educational intervention, only $24.36 \%$ of respondents correctly identified that antibiotics do not work on most cold \& cough. Fortunately, after receiving some didactic educational intervention the number of respondents who became correctly answered this question increased highly into $73.88 \%$. The same thing

Table 1. Profile of knowledge before and after intervention.

\begin{tabular}{lrr}
\hline \multicolumn{1}{c}{ Knowledge on antibiotic use and resistance (N=268) } & Pre-intervention & Post-intervention \\
\cline { 2 - 3 } & $\mathbf{N}(\mathbf{\%})$ & $\mathbf{N}(\boldsymbol{\%})$ \\
\hline Indication of antibiotics & $65(24.36)$ & $198(73.88)$ \\
\hline Antibiotics work on most cold \& cough & $87(32.57)$ & $156(58.21)$ \\
Antibiotics can treat a sore throat & $160(59.70)$ & $241(90.14)$ \\
Antibiotics can kill bacteria & $58(21.55)$ & $177(66.04)$ \\
Antibiotics can be used to treat viral infections & & \\
\hline Access to antibiotics & $206(76.88)$ & $265(98.88)$ \\
\hline Antibiotics can be purchased without prescription & $116(43.24)$ & $154(57.46)$ \\
Leftover antibiotics are good to keep at home in case of future need & & \\
\hline Administration of antibiotics & $145(54.10)$ & $255(95.15)$ \\
\hline If you feel better you can stop taking antibiotics & $89(33.00)$ & $145(54.10)$ \\
Taking less antibiotic than prescribed is healthier than taking the full course & & \\
prescribed & & $178(66.42)$ \\
\hline Antibiotic resistance & $123(44.78)$ & $134(50.00)$ \\
\hline Overuse of antibiotics can cause antibiotic resistance & $62(23.10)$ & $132(49.25)$ \\
Antibiotic resistance occurs when the body becomes resistant to antibiotics & $52(19.33)$ & \\
Antibiotic resistance is only a problem for people who take antibiotics & & \\
regularly & & \\
\hline
\end{tabular}

goes to the second question where only $32.57 \%$ of respondents in the pre-interventional phase agreed that antibiotics cannot treat a sore throat. Whereas, after education the number was also increasing into $58.21 \%$ respondents. Almost all of respondents $(90.14 \%)$ finally became aware that antibiotics is intended to kill bacteria after accepting didactical education about appropriate antibiotics use. More than half of respondents (66.04\%) also finally knew that antibiotics cannot be used to treat viral infections as a result of the education afterwards.

A vast majority of respondents had misconception about antibiotics access where $76.88 \%$ 
of them incorrectly believed that antibiotics can be purchased without prescription. It is fortunate that didactic educational intervention from pharmacists given to respondents had succesfully made 98.88\% of them understood that purchasing antibiotics with prescription is a mandatory thing to do. Post-intervention, the number of respondents who eventually aware that leftover antibiotics are not good to keep at home even because of future need became slightly higher $(57.46 \%)$ than the pre-interventional phase (43.24\%). Educational intervention also made a big impact in respondents understanding towards the appropriate use of antibiotics since $95.15 \%$ of them became conscious that they should not stop taking antibiotics although they feel better before the actual duration of therapy is over.

There was also a slight improvement showed after intervention in participants' knowledge where $54.10 \%$ respondents had become understood that they should taking the full course prescribed antibiotics for a better outcome rather than taking less antibiotics prescribed. After intervention, a number of respondents who were aware that overuse of antibiotics can cause antibiotic resistance became larger (66.42\%) than before education $44.78 \%$. Half of respondents $(50 \%)$ correctly identified that antibiotic resistance does not occur when the body becomes resistant to antibiotics after receiving education. There were only a few respondents in pre-interventional phase (19.33\%) who knew that antibiotic resistance is not only a problem for people who take antibiotics regularly. This number was increasing after intervention into $49.25 \%$ of respondents.

The next part of survey in this study was targeted to assess respondents' profile of antibiotic practice before and after didactic educational intervention was given. Of the respondents, $34.76 \%$ had a good practice even before receiving the intervention showed by their confession in taking antibiotics only for infection, meanwhile after intervention more than half of respondents (76.49\%) became aware with this issue. A large number of respondents $(78.09 \%)$ already implemented appropriate practice of antibiotics by gotten antibiotics from prescription and fortunately after intervention this number was increasing into $91.42 \%$. A magnificent result was also found in this study after intervention where almost all of respondents $(98.51 \%)$ became fully aware that taking full dose of antibiotics prescribed was also a form of appropriate antibiotics use. Respondents also showed a better understanding by declared that they had become more cautious about antibiotics use after accepting didactic educational intervention $(92.61 \%)$. Overall, Pharmacist-initiated intervention on educating respondents has made most of them more knowledgeable so hopefully they can correct their practice in antibiotics usage (Table 2)

Generally, the results of this study showed a good improvement in respondents' knowledge and practice regarding antibiotics usage as a result of implemented-educational intervention. For each domain, the number of respondents switched to a better knowledge were refined (Table 3). The highest improvement was found in the domain of antibiotic practice which made $92.26 \%$ of respondents perceived a better cognizance about appropriate practice of antibiotics. In contrary, the domain of antibiotic resistance gained only $55.22 \%$ improved-respondents after

Table 2. Profile of antibiotic practice.

\begin{tabular}{lcc}
\hline \multicolumn{1}{c}{ Practice of antibiotics usage } & Pre-intervention & Post-intervention \\
\cline { 2 - 3 } & $\mathbf{N}(\%)$ & $\mathbf{N}(\%)$ \\
\hline I am taking antibiotics only for infection & $93(34.76)$ & $205(76.49)$ \\
I got antibiotics from prescription & $211(78.09)$ & $245(91.42)$ \\
I am taking full dose of antibiotics prescribed & $186(69.32)$ & $264(98.51)$ \\
I have become more cautious about antibiotics use & $86(32.19)$ & $255(92.61)$ \\
\hline
\end{tabular}

Table 3. Domain of knowledge and practice before and after intervention.

\begin{tabular}{lccl}
\hline \multirow{2}{*}{ Variable } & Pre-intervention & Post-intervention & \multirow{2}{*}{$\mathbf{N}(\%)$} \\
\cline { 2 - 3 } Knowledge & $\mathbf{N}(\%)$ & & $<0.001^{*}$ \\
\hline Indication & & $173(64.46)$ & \\
Access to antibiotics & $113(41.75)$ & $154(78.17)$ & \\
Administration & $161(60.00)$ & $145(74.63)$ & \\
Antibiotic resistance & $117(43.55)$ & $148(55.22)$ & $<0.001^{*}$ \\
\hline Practice & $79(29.00)$ & $247(92.26)$ & \\
\hline
\end{tabular}

*significant at $p<0.05$ (Wilcoxon rank test) 
intervention which was the lowest among all domains of the questionnaire.

The level of knowledge about antibiotics use were classified as poor, adequate, and high measured in both pre and post-educational intervention. Practice of antibiotics usage was also assessed before and after intervention. The comparison of the results from each group in 2 different phases was presented in Figure 1.

Increased number of more knowledgeable respondents were showed in each group after accepting intervention. It is good to know that respondents with high knowledge of antibiotics use and resistance was already the biggest number compared with another poor and adequate groups even before intervention (37.43\%).

This number was increasing into $49.25 \%$ after intervention followed by another group which also got better in adequate group (38.28\%). The intervention also likely to reduce the number of respondents in poor knowledge group (12.21\%) and switched them to a better level of knowledge. Another good thing reported in this study was a result which showed more than half of respondents $(55 \%)$ already done appropriate antibiotics practice before intervention, added by other respondents in post-interventional phase which made a total of $75.24 \%$ respondents had

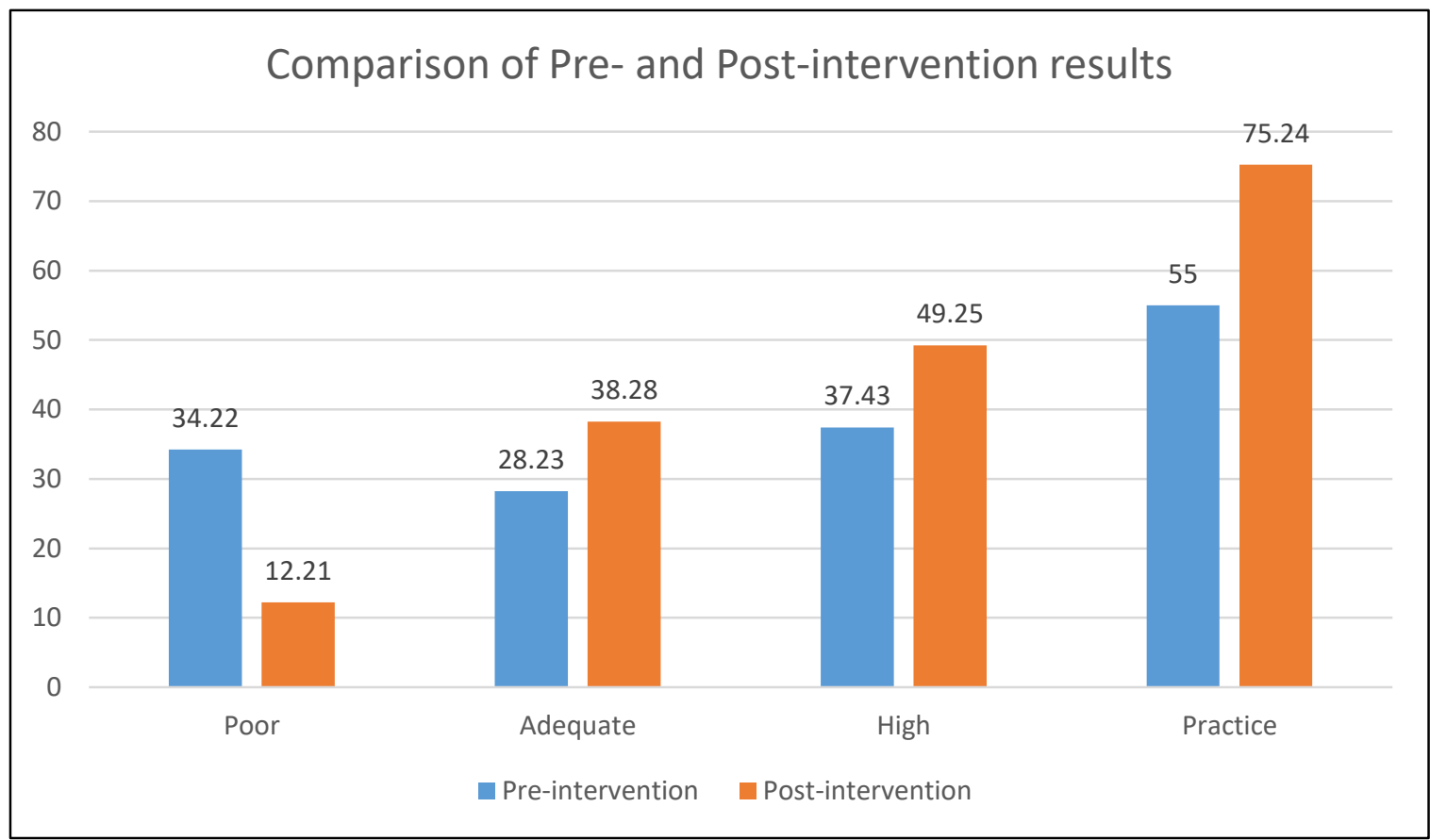

Figure 1. Comparison of Pre- and Post-intervention results

done appropriate practice of antibiotics usage.

\section{DISCUSSION}

This study emphasized the effect of didactic educational intervention about appropriate antibiotics use and showed that this kind of strategy can be very useful and effective to improve public knowledge and practice towards antibiotics use. The results of this study were in line with previous surveys worldwide that pharmacist-initiated intervention notably influenced public knowledge of appropriate antibiotics use and antibiotic resistance phenomenon ${ }^{17-19}$. The intervention applied in this study was able to shift respondents' misconception about indication of antibiotics. Before intervention, only $21.55 \%$ respondents knew that antibiotics do not work on viral infection and then after intervention this number was increasing quite high into half of respondents (50\%). This result was supported by a study conducted in Portugal which showed that educational intervention significantly affects participants' knowledge of the correct use of antibiotics for bacterial diseases rather than viral diseases $^{20}$.

Pre-intervention, more than half of respondents $(76.88 \%)$ successfully identified the appropriate access to antibiotics manifested by their correct response that antibiotics cannot be purchased without prescription. An impressive enhancement was found in this study where almost of all respondents (98.88\%) finally realized that purchasing antibiotics should escorted by a 
a valid prescription. These findings showed the importance of educating antibiotics access regulation especially in Indonesia written in Ministry of Health Law no 8 year 2015 about the action plans on antimicrobial resistance. Prescription requirement policy can be a very useful model for a country in South East Asia like Indonesia as suggested by a systematic review about government policy intervention worldwide in order to improve publics' knowledge and practice regarding appropriate antibiotics use ${ }^{21}$.

The findings also showed that $54.1 \%$ respondents before intervention already understood that they cannot stop taking antibiotics although they feel better. In the post-interventional phase, this number elevated highly into 95.15\%. Another good result regarding respondents' knowledge on antibiotics administration was showed by a slight improvement as a result of intervention in respondents' awareness that they should taking the full course antibiotic prescribed $(54.10 \%)$. These results can be interpreted that educational intervention given by pharmacists can evaluate participants' knowledge about proper antibiotics administration. This is consistent with a study conducted in Poland that showed the positive effect of educational intervention on increasing the percentage of people who have become more disciplined when using antibiotics and pay attention to the correct dosage of antibiotics prescribed ${ }^{22}$.

Regarding antibiotic resistance issues, respondents' knowledge slightly elevated after accepting educational intervention. Preintervention, $44.78 \%$ respondents already aware that overuse of antibiotics can cause antibiotic resistance. This number was increasing because of the implemented intervention into $66.42 \%$. Educational intervention also showed a good impact in correcting participants' misconception about antibiotic resistance that does not occur when the body becomes resistance to antibiotics from pre-intervention (23.1\%) to post-intervention (66.42\%). According to a previous study also held in Yogyakarta, an intervention such as antibiotics stewardship program provided by pharmacists is a good method to control antibiotic resistance phenolmenon and improve the quality of patient care $^{23}$. The results in present study reveal a potential strategy to improve patient health outcome by using pharmacist-initiated educational intervention.

This study also found that didactic educational intervention given by pharmacists notably able to raise majority of respondents' practice regarding antibiotics use. Majority of respondents in this study became thoroughly aware about appropriate practice of antibiotics $(92.26 \%)$. These findings were in line with previous similar study conducted in Jordan that highlighted a remarkable improvement in the level of knowledge of safe and also appropriate use of antibiotics caused by active educational education from pharmacist ${ }^{19}$. Improvement on participants' practice of antibiotics use indicated that educational campaigns in Indonesia are needed and hopefully be implemented as a good behavior towards antibiotics use.

Pharmacists-initiated educational intervention showed a positive impact towards participants' knowledge and practice of antibiotics usage in this study. A number of participants became more knowledgeable increase in each adequate and high knowledge group, while decrease in the poor knowledge category. However, the reality that poor knowledge group is still exist even with only few respondents as the members support the need for comprehensive educational intervention in this community. In addition, our earliest study before also in Yogyakarta highlighted that elderly individuals, male, lower level of education than bachelor, and those who rely on the internet as the source of information about antibiotics use had the most limited knowledge of antibiotics use $^{16}$. Thus, didactic educational intervention should be attentively designed and focused to reach the vulnerable groups as the priority then developed to the wider scale covering all members of community. Further evidence also showed that educational intervention from pharmacists could strengthen the programs towards preventing unnecessary and irrational use of antibiotics by: promoting adherence to appropriate prescribing guidelines, decreasing demand on antibiotics viral upper respiratory infections and increasing adherence to prescribed antibiotics ${ }^{24}$.

To sum up, this study presents the important of pharmacists-initiated educational intervention in helping the community raise their knowledge and correct their practice towards antibiotics use. This conclusion was supported by several systematic reviews which stated that educational interventions focused on appropriate antibiotics use can be successful accompanied with comprehensive analysis of local context and barriers ${ }^{10,11}$. However, single interventions targeted to public would need 
another support called multifaceted interventions which involve not only the public but also healthcare professionals by active and passive education. Moreover, our previous study also supported this kind of intervention to be implemented since $78.4 \%$ of the study population mentioned healthcare professional as their source of information, but their confession were not completely associated with good knowledge of antibiotics ${ }^{15}$.

Potential limitations in our study methods and procedure include the lack of respondents' demographic information which cause our incapability to fully describe the sample and determine the efficacy of the intervention among specialized individual subtypes. These findings may not be able to generalized the whole population or another setting of health care since it was conducted in a local area of Indonesia. Further studies on a larger scale was required to validate the result by involving the role of multifaceted intervention. Regardless the limitations identified, our findings are important to navigate a better knowledge and practice towards antibiotics use in a community setting.

\section{CONCLUSIONS}

Pharmacists-initiated educational interventions positively improve public's knowledge and practice regarding appropriate use of antibiotics in Yogyakarta. Further systematic interventions involving another healthcare professional are really needed to reach sustainable improvement of public's attitude towards antibiotics use.

\section{ACKNOWLEDGMENT}

We especially thank the participants for their kindness to fulfill the questionnaire. We also thank pharmacists in Yogyakarta province.

\section{Funding}

None to declare

\section{Conflict of interest}

Authors declared no conflict of interest in this study

\section{Ethical approval}

This study protocol was approved by Medical and Health Research Ethics Committee, Universitas Gadjah Mada, Yogyakarta.

\section{Article info:}

Received May 11, 2020

Received in revised form August 10, 2020

Accepted October 25, 2020

\section{REFERENCES}

1. Cars O, Högberg LD, Murray M, Nordberg O, Sivaraman S, Lundborg CS, et al. Meeting the challenge of antibiotic resistance. BMJ. 2008;18(337).

2. World Health Organization. Global action plan on antimicrobial resistance. Geneva: World Health Organization, 2015

3. Courvalin P. Predictable and unpredictable evolution of antibiotic resistance. J Intern Med. 2008;264(1):4-16.

4. Davies J, Davies D. Origins and evolution of antibiotic resistance. Microbiol Mol Biol Rev. 2010;74(3):417-33.

5. Altiner A, Brockmann S, Sielk M, Wilm S, Wegscheider K, Abholz HH. Reducing antibiotic prescriptions for acute cough by motivating GPs to change their attitudes to communication and empowering patients: a cluster-randomized intervention study. J Antimicrob Chemother. 2007;60(3):638-44.

6. Arroll B, Kenealy T, Kerse N. Do delayed prescriptions reduce the use of antibiotics for the common cold? A single-blind controlled trial. J Fam Pract. 2002;51(4):324-8.

7. Wood F, Phillips C, Brookes-Howell L, Hood K, Verheij T, Coenen S, et al. Primary care clinicians' perceptions of antibiotic resistance: a multi-country qualitative interview study. J Antimicrob Chemother. 2013;68(1):237-43.

8. Fridkin SK, Srinivasan A. Implementing a strategy for monitoring inpatient antimicrobial use among hospitals in the United States. Clin Infect Dis. 2014;58(3):401-6.

9. Yong MK, Buising KL, Cheng AC, Thursky KA. Improved susceptibility of Gram-negative bacteria in an intensive care unit following implementation of a computerized antibiotic decision support system. J Antimicrob Chemother. 2010;65(5):1062-9.

10. Arnold SR, Straus SE. Interventions to improve antibiotic prescribing practices in ambulatory care. Cochrane Database Syst Rev. 2005;19(4).

11. Ranji SR, Steinman MA, Shojania KG, Gonzales R. Interventions to reduce unnecessary antibiotic prescribing: a systematic review and quantitative analysis. Med Care. 2008;46(8):847-62.

12. Parathon H, Kuntaman K, Widiastoety TH, Muliawan BT, Karuniawati A, Qibtiyah M, et al. Progress towards antimicrobial resistance containment and control in Indonesia. BMJ. 2017;358:j3808.

13. Kurniawan K, Posangi J, Rampengan N. Association between public knowledge regarding antibiotics and self-medication with antibiotics in Teling Atas Community Health Center, East Indonesia. Med J Indones. 2017;26(1):62-9.

14. Widayati A, Suryawati S, de Crespigny C, Hiller JE. Beliefs about the use of nonprescribed antibiotics among people in Yogyakarta City, Indonesia: a qualitative study based on the theory of planned behavior. Asia Pac J Public Health. 2015;27(2):1.

15. Salsabila NN, Kristina SA. Awareness on antibiotic resistance among lay people in Yogyakarta, Indonesia. Int J Pharm Res. 2020;12(3):180-8.

16. Kristina S, Wati R, Prasetyo S. Public knowledge and awareness towards antibiotics use in Yogyakarta: A 
cross sectional survey. Pharm Sci Asia. 2020;47(2):174-81.

17. Ashiru-Oredope D, Hopkins S. Antimicrobial resistance tance: moving from professional engagement to public action. J Antimicrob Chemother. 2015;70(11):2927-30.

18. Huttner B, Goossens H, Verheij T, Harbarth S. Characteristics and outcomes of public campaigns aimed at improving the use of antibiotics in outpatients in high-income countries. Lancet Infect Dis. 2010;10 (1):17-31.

19. Shehadeh MB, Suaifan GA, Hammad EA. Active educational intervention as a tool to improve safe and appropriate use of antibiotics. Saudi Pharm J. 2016;24 (5):611-5.

20. Azevedo MM, Pinheiro C, Yaphe J, Baltazar F. Assessing the impact of a school intervention to promote students' knowledge and practices on correct antibiotic use. Int J Environ Res Public Health.
2013;10(7):2920-31.

21. Rogers Van Katwyk S, Grimshaw JM, Nkangu M, Nagi R, Mendelson M, Taljaard M, et al. Government policy interventions to reduce human antimicrobial use: A systematic review and evidence map. PLoS Med. 2019;16(6):e1002819.

22. Mazińska B, Hryniewicz W. European Antibiotic Awareness Day educational campaign--has it changed public attitudes to antibiotic use in Poland?. Pol Merkur Lekarski. 2010;29(173):296-303.

23. Asvingita LRM, Sari IP, Kristina SA. Antibiotics stewardship practice among community pharmacists in Indonesia: A cross-sectional survey. Int J Pharm Res. 2020;11(4):176-81.

24. Suda KJ, Wiskirchen DE, Advincula R. Improving safe and appropriate use of antibiotics. J Am Pharm Assoc. 2012;52:6-10. 